\title{
Integración de Lean, \\ Design Thinking y Agile en la gestión de proyectos*
}

\author{
Integration of Lean, Design Thinking and Agile \\ in project management
}

\section{Inteǵração do Lean, do Design Thinking e do Agile na gestão de projetos}

Edwin Orlando Arias Bareño"

\section{RESUMEN}

La gestión de proyectos es actualmente un amplio campo de investigación dada la diversidad de metodologías y marcos de trabajo que surgen día a día en diferentes áreas de conocimiento, por lo que el presente documento propone una integración de tres marcos de trabajo que son tendencia en diferentes campos. Lo anterior tiene lugar a partir de una revisión literaria de 22 artículos alojados en las bases de datos Science Direct y
Scopus principalmente, los cuales fueron escogidos mediante una depuración sistemática con base en criterios previamente definidos. Seguido a ello, se realiza una definición de los conceptos proyecto, gestión de proyectos y metodología de gestión de proyectos, lo que a su vez permitió reconocer la importancia de las oficinas de gestión de proyectos en el ámbito organizacional, y el papel que estas juegan para permear y enseñar en las compañías.

Artículo de resultado de reflexión

** Especialista en Alta Gerencia, Ingeniero Industrial. Consultor en Gestión de Proyectos - Gerencia de Innovación y Transformación Digital, Porvenir S.A. Correo electrónico: edwinarias622@gmail.com. oRCID: https://orcid.org/0000-0002-7463-4087 
Se realizó una aproximación al concepto y principales características de design thinking, orientada a la creación y diseño a partir de la experimentación con el usuario; lean, que pretende optimizar y reducir desperdicios, y agile, que reúne aspectos de las dos anteriores, con la definición de mínimo producto viable y priorización de funcionalidades con el usuario. Finalmente, se propuso un marco de trabajo que reúne las principales características y buenas prácticas de estas tres, concluyendo así en un modelo adaptable desde la oficina de gestión de proyectos para implementar en cualquier organización.

Palabras clave: agile, design thinking, gestión de proyectos, lean, metodologías de proyectos, oficina de gestión de proyectos.

\section{ABSTRACT}

Project management is currently a broad field of research given the diversity of methodologies and frameworks that emerge every day in different areas of knowledge. Therefore, this document proposes an integration of three frameworks that are trends in different fields. This is based on a literature review of 22 articles, mainly from Science Direct and Scopus, which were chosen through a systematic selection based on previously defined criteria. Then, the project, project management and project management methodology concepts were defined, which in turn led to recognize the importance of Project Management Offices in the organizational environment, and their role in permeating and teaching in companies. An approach was made to the concept and main characteristics of Design Thinking, oriented towards creation and design based on experimentation with the user, Lean, which aims to optimize and reduce waste, and Agile, which brings together aspects of the two previous ones, with the definition of a minimum viable product and prioritization of functionalities with the user. Finally, a framework was proposed that brings together the main characteristics and good practices of these three, thus concluding in a model adaptable from the Project Management Office to be implemented in any organization.

Keywords: Project management, project methodologies, Design Thinking, Lean, Agile, Project Management Office.

\section{RESUMO}

A gestão de projetos é hoje um campo amplo de pesquisa em virtude da diversidade de metodologias e quadros que surgem a cada dia em diferentes áreas do conhecimento, pelo que este documento propõe uma integração de três quadros que são tendências em diferentes áreas. O acima exposto surgiu a partir de uma revisão bibliográfica de 22 artigos, principalmente Science Direct e Scopus, que foram escolhidos através de uma purificação sistemática baseada em critérios previamente definidos. Seguiu-se uma definição dos conceitos de projeto, gestão de projetos e metodologia de gestão de projetos, o que por sua vez levou ao reconhecimento da importância dos Escritórios de Gestão de Projetos no ambiente organizacional e do papel que desempenham para permear e ensinar nas empresas. Foi feita uma reflexão sobre o conceito e os principais características do Design Thinking, voltado para a criação e design a partir da experimentação com o usuário, do Lean, que tenta otimizar e reduzir desperdícios, e do Agile, que reúne dimensões dos dois anteriores, com a definição de produto mínimo viável e priorização de funcionalidades com o usuário. Por fim, foi proposto um quadro que reúne as principais características e boas práticas destes três, concluindo, assim, com um modelo adaptável pelo Escritório de Gestão de Projetos para ser implementado em qualquer organização. 
Palavras-chave: Gestão de projetos, metodologias de projetos, Design Thinking, Lean, Agile, escritório de gestão de projetos.

\section{INTRODUCCIÓN}

La gestión de proyectos actualmente es un factor clave en el éxito de las organizaciones, por lo que recientemente se ha incrementado el interés en definir su razón de ser. Pero ¿̨cuál es la diferencia entre gestión de proyectos y gestión de empresas?; la respuesta es que la gestión de proyectos es esencialmente la gestión del cambio, mientras que la gestión de un negocio funcional o empresa es la gestión de un proceso continuo o habitual (Lester, 2017). En busca de una gestión eficiente de los proyectos, y difundir estas buenas prácticas al mundo, se han generado múltiples metodologías y marcos de trabajo para su gestión y la de sus portafolios, relacionadas, por ejemplo, al sector económico o al tipo de proyecto.

La literatura de gestión de proyectos distingue entre formas de gestión de proyectos estandarizadas y personalizadas (Joslin \&Müller, 2015); recientemente, se incorporaron las prácticas de gestión ágiles, que proporcionan un conjunto de buenas prácticas que permiten adaptaciones rápidas coincidentes con las necesidades modernas de desarrollo de productos (Papadopoulos, 2015). Si bien en principio esto surgió como respuesta a una necesidad en la gestión de proyectos de desarrollo de software, se ha integrado con otras metodologías generando buenos resultados en otros ambientes, gracias a sus prácticas de interacción permanente y alta respuesta al cambio.

En el 2008, Lean era tendencia y la metodología mayormente utilizada por su funcionalidad, no solo para la gestión de proyectos, sino también en la gestión de empresas y negocios. Lean se ha considerado como una metodología de mejora empresarial que integra dos filosofías de gestión distintivas: Lean y Six-Sigma, que se complementan entre sí para mejorar los procesos y resultados de las empresas (Tenera \& Pinto, 2014).
Otra metodología ampliamente utilizada principalmente en el diseño de productos, pensando primero en la experiencia del usuario, es Design Thinking. Una de las áreas desplegadas en las teorías y prácticas de gestión es la aplicación más amplia del diseño y el pensamiento de diseño (Design Thinking) en estrategias y modelos de negocios para crear valor para los clientes y aumentar el valor para la organización en sí(Volkova \& Jãkobsone, 2016).

Lean y Design Thinking, cada una independientemente, se han integrado con Agile; por ejemplo, en el uso de un enfoque Design Thinking que promueve la comunicación entre los equipos ágiles de desarrollo de software y los clientes a lo largo de todo el proyecto de desarrollo del producto (Pereira \& Russo, 2018). Sin embargo, no se ha encontrado dentro de la literatura revisada la integración de Lean, Design Thinking y Agile para un mismo propósito.

Las organizaciones se enfrentan a mercados competitivos y globalizados, así como a constantes cambios ambientales que a menudo requieren una reestructuración organizativa de los modelos de negocios para impulsar el desempeño. Las prácticas de gestión de proyectos pueden ayudar a alcanzar objetivos estratégicos y aumentar el valor de los proyectos en las organizaciones (Monteiro, Santos, \& Varajão, 2016). La oficina de gestión de proyectos (PMO) es una estructura organizativa creada con el fin de promover y mejorar la gestión de proyectos, mediante la adopción de metodologías, prácticas y marcos de trabajo adecuadas para lograr altos niveles de eficiencia y eficacia (Monteiro et al., 2016). La investigación sobre las PMO se ha concentrado en su estructura y función como integrador para facilitar, coordinar y respaldar la actividad de proyectos en organizaciones y carteras (Paton \& Andrew, 2019). Es importante evaluar la funcionalidad de un marco de trabajo para la gestión de proyectos en empresas, que integre bondades de Design Thinking, Lean y Agile, apalancando esta evaluación desde la PMO. 
Se debe partir primero de la definición del concepto actual de proyectos en un contexto general, por lo que se puede afirmar que un proyecto es un proceso único, que consiste en un conjunto de actividades coordinadas y controladas con fechas de inicio y finalización, realizado para lograr objetivos que se ajusten a los requisitos específicos, incluidas las limitaciones de tiempo, costo y recursos (Lester, 2017). En segundo lugar, se debe establecer el conocimiento de las metodologías Design Thinking, Lean y Agile, y sus campos de aplicación organizacional más comunes. Finalmente, es preciso proponer una integración apalancada desde PMO de Lean, Design Thinking y Agile para la gestión de proyectos en empresas.

En busca de generar un marco de trabajo general y de utilidad en la gestión de proyectos en las empresas, dada la alta variabilidad del mercado actual, se realiza una revisión de literatura mediante la identificación de palabras clave y autores reconocidos en siete (7) variables de investigación definidas. Se identifica un concepto general de proyecto y sus metodologías de gestión, presentando también el estado actual de las formas de trabajo estandarizadas Lean, Design Thinking y Agile, y algunas de sus integraciones en casos reales como Lean and Agile Performance Framework para empresas fabricantes (Soltan \& Mostafa, 2015), y Design Thinking integrado en el desarrollo de software ágil (Pereira \& Russo, 2018). Finalmente, se propone una buena práctica personalizada para la gestión de proyectos que integra lo mejor de Lean, Design Thinking y Agile.

\section{METODOLOGÍA DE INVESTIGACIÓN}

\section{Estrategia de búsqueda}

Teniendo en cuenta que la gestión de proyectos es investigada permanentemente en todo el mundo por empresas y universidades, existe un amplio espectro de búsqueda sobre el tema, por lo que se acotó a bases de datos reconocidas por el estudio de ciencias administrativas e ingeniería industrial (Science Direct, Scopus, IEEE xplore, Redalyc), y documentos con fecha de publicación no mayor a cinco años.

Uno de los criterios para la obtención de una muestra razonable que contribuya al cumplimiento del objetivo de este estudio fue la integración de las siguientes palabras clave: Project Management Office, Agile, Lean y Design Thinking, como complemento a la búsqueda principal relacionada con el término en inglés Management Project Methodology. Posteriormente, se realizó la aplicación de filtros de razonabilidad, principalmente de un tipo de documento: artículos de investigación, y se realizó la exclusión de artículos muy específicos en cuanto a casos de estudio orientados a manufactura y ciencias de la salud.

\section{Búsqueda de literatura}

Se definen seis (6) variables de investigación: 1) definición de proyecto, 2) metodologías en el desarrollo de proyectos, 3) metodología Design Thinking, 4) metodología Agile, 5) metodología Lean y 6) integración entre metodologías (tabla 1). En conjunto, con base en estas seis variables y la ecuación de búsqueda creada a partir de los criterios definidos, se indaga en cada una de las bases de datos seleccionadas.

Ecuación de búsqueda: TITLE-ABS-KEY (( management AND project AND methodology ) OR (agile) ) AND ( LIMIT-TO ( DOCTYPE, "ar")) AND ( LIMIT-TO ( PUBYEAR, 2019 ) OR LIMIT-TO ( PUBYEAR , 2018 ) OR LIMIT-TO ( PUBYEAR, 2017 ) OR LIMIT-TO ( PUBYEAR, 2016 ) OR LIMIT-TO (PUBYEAR, 2015)) 
Tabla 1. Palabras clave adicionales a ecuación de búsqueda según variable de investigación

\begin{tabular}{|l|l|}
\hline \multicolumn{1}{|c|}{ Variables de investigación } & \multicolumn{1}{c|}{ Palabras clave adicionales de búsqueda } \\
\hline Definición de Proyecto & Definition of Project OR Enterprise Projects \\
\hline Metodologías en el Desarrollo de Proyectos & Projects Methodologies OR Project Management Office \\
\hline Metodología Design Thinking & Design Thinking OR Customer Service Projects \\
\hline Metodología Lean & Lean Startup OR Lean Six Sigma \\
\hline Metodología Agile & SCRUM OR Development Software \\
\hline Integración de Metodologías & (Agile AND Lean) OR (Agile AND Design) \\
\hline
\end{tabular}

Fuente: elaboración propia.

Figura 1. Proceso selección artículos objeto de estudio.

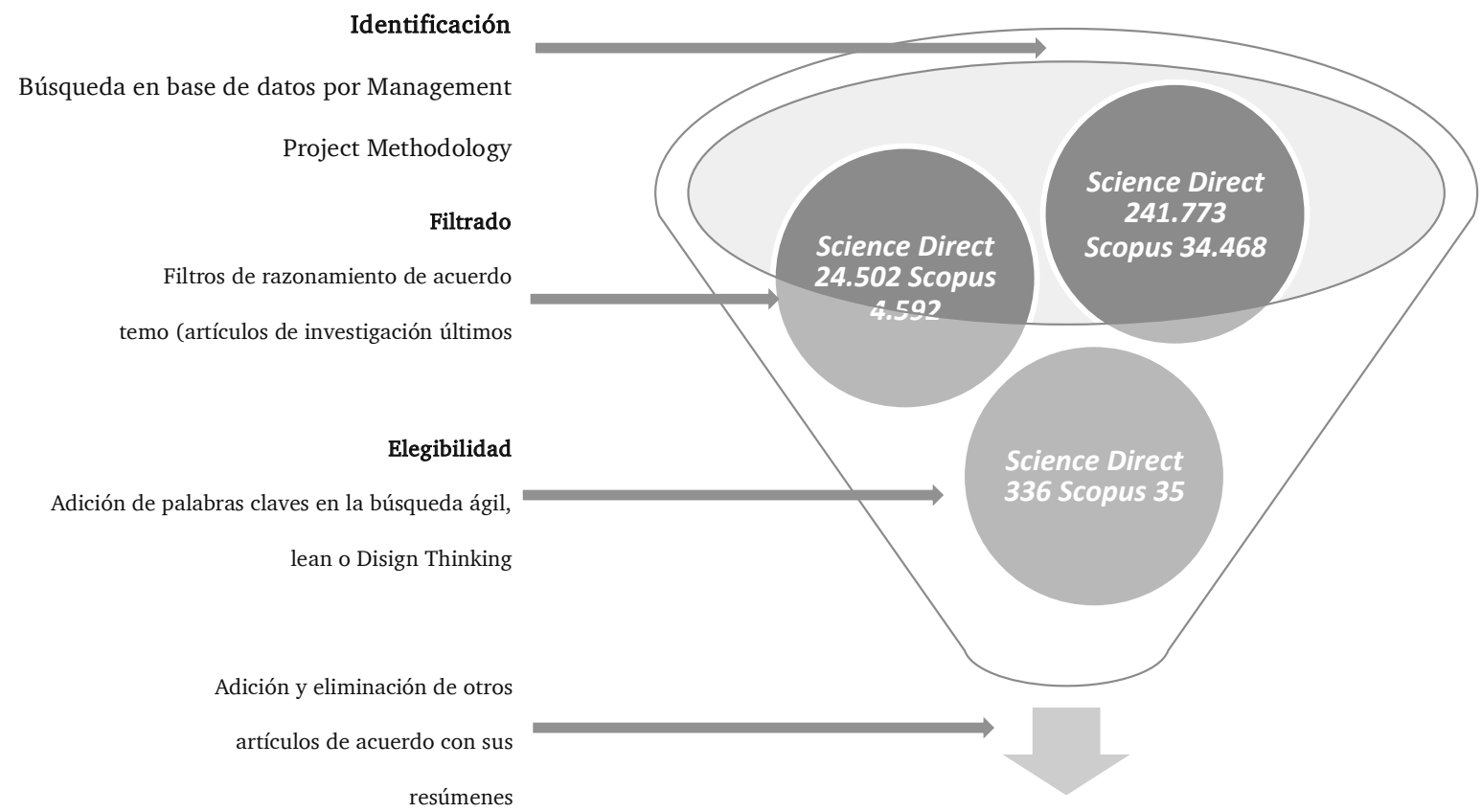

23 Articulos de investigacion incluidos resultados de revision

Fuente: elaboración propia.

La figura 1 muestra obtenidos en los procesos de selección de los artículos objeto de estudio. Identificación, filtrado y elegibilidad, procesos que arrojaron un total de 23 artículos de investigación. Por otro parte, se realizó análisis mediante la herramienta Vosviewer, incluyendo los 23 artículos en un proceso de identificación de palabras con mayor repetición dentro de los títulos, abstract y keywords; de este se determina que la muestra es afín a los objetivos y las variables definidas, dado que dentro de las palabras con mayor repetición se encuentran Project, Design Thinking, Lean, Agile Methodology y Project Management Methodology. 
Un factor fundamental para la obtención de una muestra objetiva fue la vinculación como criterio de búsqueda únicamente de publicaciones en los últimos cinco años y documentos en inglés, en donde, de acuerdo con búsquedas previas, se encuentra el pareto de la literatura entorno a metodologías de gestión de proyectos.

\section{RESULTADOS Y DISCUSIÓN}

\section{Concepto actual de gestión de proyectos en un contexto global}

\section{Proyecto}

La literatura revisada define proyecto como un proceso único que consiste en un conjunto de actividades coordinadas y controladas con fecha de inicio y finalización, realizado para lograr objetivos previamente definidos, y con limitación de recursos, tiempo y costos (Lester, 2017). Un proyecto debe cumplir con tres criterios fundamentales: 1) debe ser completado a tiempo, 2) debe ser realizado dentro del costo presupuestado y 3 ) debe cumplir con los requisitos de calidad prescritos en el alcance (Lester, 2017), lo que es considerado como la triple restricción, y lo que da origen a la gestión de proyectos (PM, por las siglas en inglés de Project
Management), como necesidad de optimizar estas restricciones.

\section{Gestión de proyectos}

La gestión de un proyecto es la planificación, organización, monitoreo y control de todos los aspectos de este, lo que se articula para lograr los objetivos de manera segura, dentro de los tiempos acordados, con el presupuesto y el alcance previamente definido. En la gestión de proyectos se centra la medición del rendimiento del proyecto, en relación con sus dimensiones a corto plazo: cumplimiento de los criterios de tiempo, costo y calidad, definidos previamente como triple restricción (Radujković \& Sjekavica, 2017).

Otra definición de gestión de proyectos ampliamente aceptada, que complementa la expuesta por Radujković y Sjekavica (2017), se refiere a que es el conjunto de procesos establecidos para organizar y administrar los recursos necesarios, para llevar a buen término el alcance definido con la calidad y el tiempo acordados, teniendo en cuenta limitaciones de recursos y costos (Samset $\&$ Volden, 2016).

Los factores en la gestión de proyectos que más contribuyen tanto al éxito de la gestión del proyecto como, en consecuencia, al éxito general del proyecto se resumen en la tabla 2.

Tabla 2. Factores críticos en la gestión de proyectos

\begin{tabular}{|l|c|}
\hline Factor en gestión de proyectos & Categoría del factor \\
\hline Competencias del gerente de proyecto & $\mathrm{CF} 1$ \\
\hline Inteligencia emocional de los gestores de proyectos, elementos de gestores de proyectos & $\mathrm{CF} 1$ \\
\hline Cosas en el equipo del proyecto. & $\mathrm{CF} 1$ \\
\hline $\begin{array}{l}\text { Aplicación de los conocimientos y habilidades de gestión de proyectos del director del proyecto y del equipo del } \\
\text { proyecto, así como su coordinación. }\end{array}$ & $\mathrm{CF1}$ \\
\hline Estructura organizativa & $\mathrm{CF2}$ \\
\hline
\end{tabular}




\begin{tabular}{|l|c|}
\hline Factor en gestión de proyectos & Categoría del factor \\
\hline Cultura organizacional & $\mathrm{CF} 2$ \\
\hline Herramientas y técnicas de gestión de proyectos. & $\mathrm{CF} 3$ \\
\hline Normas de gestión de proyectos & $\mathrm{CF3}$ \\
\hline
\end{tabular}

Fuente: adaptada de Radujković y Sjekavica (2017).

Un breve análisis de estos factores los puede resumir en tres categorías que toda gestión de proyectos debe tener en cuenta, como se destaca en la segunda columna de la tabla 2: Competencias para gestión de proyectos (CF1), Elementos de la organización (CF2) y Elementos de metodologias de gestión de proyectos, métodos, herramientas y técnicas (CF3) (Radujković \& Sjekavica, 2017).

El control en la gestión de proyectos se refiere a los procesos, sistemas y regulaciones que deben estar presentes para garantizar su éxito. Por lo general, esto incluye un marco regulatorio para garantizar una calidad adecuada, como es el caso de proyectos de tecnología como la Norma Iso 20000, el cumplimiento de los objetivos acordados, la gestión y la resolución de los problemas que puedan surgir durante el curso del proyecto, y los estándares para la revisión de la calidad de los documentos clave de evaluación (Samset \& Volden, 2016).

La gestión efectiva de proyectos es más que una gestión orientada a la ejecución. Los proyectos deben perseguir el objetivo de crear valor y entregar beneficios, dando sentido a la interacción entre los objetivos del sponsor o dueño del proyecto y su desarrollo de una manera óptima; este es probablemente uno de los aspectos más importantes en la gestión de un proyecto (Morris, 2009).

Si bien la literatura sobre gestión de proyectos es muy relevante, hasta hace poco se convirtió en un tema de interés e importancia para la comunidad investigadora (Samset \& Volden, 2016), surgiendo de esta manera el concepto de PMO.

\section{Oficina de gestión de proyectos (PMO)}

La PMO es una estructura organizativa creada para promover y mejorar la práctica en la gestión de proyectos, mediante la adopción de distintas metodologías apropiadas para lograr altos niveles de eficiencia y eficacia (Monteiro et al., 2016).

La gestión de proyectos es ineludible en el mundo de hoy, un lugar de mejora continua. La gestión de proyectos no solo es una necesidad para esa mejora, sino también un campo que busca la mejora en sí misma, a través de la influencia que puedan ejercer este tipo de figuras como la PMO y la correcta integración de metodologías que apliquen. Por eso, es importante promover la competencia, el conocimiento y las mejores prácticas a través de este organismo. La parte significativa de esta educación debe ser interna organizativa, es decir, que permee toda la organización (Radujković \& Sjekavica, 2017).

La necesidad de una respuesta oportuna a los cambios del mercado, la demanda de los clientes y cada vez más rápidas mejoras tecnológicas llevan a las organizaciones a desarrollar sus habilidades por medio de una correcta articulación de proyectos que generen valor a partir de nuevas funcionalidades para el cliente.

El análisis contemporáneo del control de proyectos dentro de grandes organizaciones con gran número de estos generalmente incluye un examen de la forma y función de la PMo; el trabajo inicial que describe a la PMO es servir 
como unidades de apoyo que llevan a cabo actividades rutinarias como la administración y el archivo (Powell \& Young, 2007); sin embargo, en un trabajo más reciente se actualiza este concepto, argumentando que las PMo se están volviendo cada vez más sofisticadas, expandiendo su actividad a mucho más que completar las tareas básicas. Un nuevo concepto propone que las PMo ejecuten tareas de soporte de proyectos de despliegue más amplio de sistemas de control y un nivel de coordinación más complejo (Paton \& Andrew, 2019).

Por todo lo anterior, el conocimiento y la comprensión de la gestión de proyectos ha crecido y las organizaciones reconocen la importancia de la gestión de proyectos para el desarrollo empresarial. Por ello, se deben realizar esfuerzos en el fortalecimiento organizacional de los proyectos, estableciendo e incorporando una nueva entidad en las empresas: la PMO, que puede aparecer sola en una organización o como múltiples PMO, de acuerdo con el tamaño de las empresas e impacto de los proyectos, encargadas de diferentes tareas de gobernabilidad de proyectos, alcance de las actividades y autoridades (Monteiro et al., 2016).

La PMO es, por lo tanto, una unidad o departamento, en organizaciones matriciales o en organizaciones basadas en proyectos, para desarrollar metodologías e institucionalizar prácticas de gestión de proyectos (Monteiro et al., 2016).

\section{Design Thinking, Lean y Agile, y sus campos de aplicación organizacional}

\section{Design Thinking (DT)}

El diseño es la conexión entre quien busca experiencias y quien trata de satisfacer ese apetito con una oferta que presenta lo nuevo de una manera fácil e innovadora; además, se entiende como el núcleo de la economía del conocimiento y uno de los retos para afrontar en los sistemas de innovación (Volkova \& Jãkobsone, 2016).
"El pensamiento de diseño es una metodología que imbuye el espectro completo de las actividades de innovación con un espíritu de diseño centrado en el ser humano" (Chou, 2018, p. 75). Si bien es necesaria una estructuración en las metodologías de la gestión de proyectos, la metodología DT no consiste en una serie de pasos ordenados, rígidos y predefinidos. DT consiste en un proceso por fases que permiten ir refinando una idea a partir de procesos de innovación (Glen, Suciu, Baughn, \& Anson, 2015).

Estudios en el mundo, como el del Centro de Diseño Danés (DDC), y la Oficina de Asociaciones de Diseño Europeas (BEDA), entre otros, demuestran que la medida en que el diseño mejora la creatividad, la innovación y la competitividad depende de la forma de implementación y despliegue que se realice en la empresa (Volkova \& Jãkobsone, 2016), es decir, en cómo se interioriza la metodología en la organización.

Las actividades de diseño en una compañía deben estar estrechamente relacionadas con los objetivos, formando tres niveles principales de uso del diseño: diseño como producto, diseño como proceso y diseño como transformación. Este último nivel se considera como la competencia del diseño en la creación de procesos de innovación (Volkova \& Jãkobsone, 2016).

Si bien la metodología DT es un refinamiento continuo de una idea, algunos autores se aventuran a estructurarla en una serie de fases, con la advertencia de que no todos los proyectos de DT se adherirán a este grado de linealidad. Un marco que sirve como guía es el conjunto básico de pasos y principios de DT: 1) localización de problemas, 2) observación, 3) visualización y creación de sentido, 4) ideación, 5) creación de prototipos y pruebas, y 6) pruebas de viabilidad (Glen et al., 2015); en estas pruebas de viabilidad es en donde surgen los hallazgos a partir de los cuales se dan los nuevos refinamientos.

Algunos principios de DT son los siguientes: la competitividad se logra creando productos con un nuevo 
significado y un mayor valor agregado, énfasis en el significado de los productos y su valor; el proceso de diseño se fusiona con los objetivos clave de las empresas; el diseño integra tecnología, funciones comerciales e identidad humana, y se deben abrir innovaciones en diversos caminos a través de la transformación de la cultura de la organización (Glen et al., 2015).

El pensamiento de diseño es un enfoque de la innovación centrado en el ser humano que se basa en el conjunto de herramientas del diseñador para integrar las necesidades de las personas, las posibilidades de la tecnología y los requisitos para el éxito empresarial. (Chou, 2018, p. 76)

\section{Lean}

Lean es una metodología implementada en la fabricación y la gestión de procesos y proyectos que ayuda a las organizaciones a mejorarlos, reduciendo e incluso eliminando el desperdicio (AlManei, Salonitis, \& Tsinopoulos, 2018). El enfoque Lean Startup ha sido un concepto revolucionario de negocio, que se recomienda implementar en empresas que quieren obtener éxito en corto plazo; es una metodología que, al igual que DT, se basa en la obtención rápida de satisfacción del cliente a través de la consulta continua. La metodología Lean Startup se define como un marco útil para reducir en gran medida el riesgo de iniciar nuevos negocios o lanzar un producto (Girgenti, Pacifici, Ciappi, \& Giorgetti, 2016).

Un concepto importante que maneja esta metodología es que, a partir de la generación permanente de ideas, se define un mínimo producto viable (MvP, por las siglas en inglés de Minimum Viable Product), que facilita y fomenta la detección de contenidos innovadores. En la definición de un MvP deben analizarse las restricciones de viabilidad, perfil de clientes y la evaluación comparativa en propuestas similares de valor de los competidores (Girgenti et al., 2016).
Existe una serie de etapas básicas en la implementación de Lean Startup basadas en sus métodos y principios: definir, medir, analizar, mejorar y controlar el ciclo (DMAIC, por sus siglas en inglés), considerado un marco de mejora continua (Tenera \& Pinto, 2014) que busca optimizar los esfuerzos en la reducción de defectos de producción (bienes o servicios) y disminuir la variabilidad del proceso, junto con la simplificación y estandarización de estos.

Los principios clave en la implementación de gestión de proyectos con metodología Lean son los siguientes: considerar la gestión del cambio desde el inicio del proyecto; contar con apoyo de la alta dirección y que exista una alineación estratégica, compromiso de gestión por parte de los interesados, la existencia tácita de una estructura organizativa, metas y objetivos, plan de transformación, seguimiento y vigilancia (AlManei et al., 2018).

Se pueden alcanzar prácticas estables a través de una identificación y evaluación continuas de oportunidades de mejora en los procesos y decisiones de gestión de proyectos, permitiendo resultados organizativos y reducción de desperdicios de procesos. La incorporación de los pasos DMAIC en cada proyecto debería ayudar a los gerentes de proyecto a ser no solo más efectivos, sino también a lograr resultados innovadores (Tenera \& Pinto, 2014).

Por lo tanto, Lean es un enfoque que permite satisfacer rápidamente, de manera eficiente y efectiva las necesidades de los clientes. En los sectores que se han aplicado, Lean Startup aceleró la consolidación de la empresa o de algún producto en el mercado; por ejemplo, el aumento de activos financieros y reducción de tiempo de desarrollo del producto. Además, este enfoque muestra a emprendedores y empresarios en poco tiempo si el nuevo negocio es bueno o no. Sin embargo, existen ejemplos de aplicabilidad en mejora en otros sectores, como en la atención médica con la implementación de nuevos canales de servicio (Silva, Calado, Silva, \& Nascimento, 2013). 


\section{Agile}

Agile es realmente la adaptación de los conceptos de Scrum, que se encuentran escritos en el manifiesto ágil, cuyos cuatro valores principales son los siguientes: 1 ) personas e interacciones sobre procesos y herramientas; 2) un producto funcionando sobre documentación extensiva; 3) colaboración con el cliente sobre negociación contractual, y 4) respuesta ante el cambio sobre seguir un plan.

Las buenas prácticas que comparten Scrum y Agile se resumen en iteraciones permanentes en ceremonias de reunión efectivas con los temas claros que se deben tratar, la definición de un MPv que genere el mayor valor, y la ausencia de un plan de trabajo a largo plazo definido, lo que permite una mayor respuesta al cambio de alcance. Lo anterior ha permitido la planificación del trabajo que debe realizar el equipo; transparencia en la evolución del trabajo; visualización y discusión de los avances de las entregas a lo largo del tiempo; dimensionamiento del esfuerzo para realizar tareas; planificación de actividades que seden desarrollar en cada sprint y reuniones; comunicación constante entre los miembros del equipo; medida clara de lo producido por el equipo y mayor calidad en las entregas (Carneiro, Silva, \& Alencar, 2018).

El hecho de contar con equipos articulados, autoorganizados y una estructura organizativa plana permite que los equipos ágiles colaboren estrechamente sin complicaciones innecesarias. En contraste con los enfoques tradicionales que dependen profundamente de la documentación y las estructuras organizativas jerárquicas, el objetivo del marco ágil es mejorar la colaboración y coordinación de los equipos y miembros para lograr una ejecución de proyectos distribuida con éxito (Papadopoulos, 2015).

Las metodologías ágiles se implementan y utilizan ampliamente en todo el mundo. La decisión de implementar o no la metodología depende de los tipos de proyectos, la empresa y sus empleados. Las características de los empleados, sus relaciones mutuas y su motivación son aspectos clave que pueden afectar seriamente el éxito de la implementación de la metodología. Por lo tanto, estos factores también deben evaluarse y considerarse durante la decisión de adaptación de la metodología (Rasnacis \& Berzisa, 2017).

Agile no es considerada por algunos como una metodología, sino como un conjunto de buenas prácticas que cada uno es libre de implementar o no. El marco ágil propone una participación basada en la iteración de todos los equipos, para lo cual, en proyectos grandes y distribuidos, el enfoque principal debe ser mantener la estructura organizativa pequeña; la jerarquía debe ser lo más plana posible, con más equipos y menos niveles de gestión (Papadopoulos, 2015).

A diferencia de Design Thinking, las fases o ciclos de esta metodología entregan un producto funcional al cliente, gracias a la priorización por valor ganado que se realizó al principio del proyecto. Las investigaciones actuales entorno a Agile incluyen análisis de características o métodos adicionales para el análisis de equipo que pueden ayudar durante la adaptación e implementación de la metodología ágil, agregando lineamientos de roles, artefactos, procesos y prácticas ágiles que se pueden usar para mejorar la autoorganización y motivación del equipo (Rasnacis \& Berzisa, 2017).

\section{Inteǵración de Desiǵn Thinking, Lean y Agile en un marco de trabajo para la
gestión de proyectos en empresas}

\section{Integración Design Thinking, Lean y Agile}

De acuerdo con las diferentes características del proyecto, Design Thinking, Lean y Agile por sí solas tienen múltiples ventajas y desventajas, por lo que la selección de una de estas debe realizarse con sumo cuidado, considerando tanto las características del proyecto como las 
características del entorno organizacional. Además, es posible realizar combinación de sus prácticas para un proyecto y dentro de un solo marco de trabajo, teniendo en cuenta cuándo es mejor usar cada una de ellas. Es importante tener en cuenta que la forma de trabajo debe adaptarse al proyecto y no al revés (Špundak, 2014). Esto último explica por qué la tendencia actual es la utilización de frameworks o marcos de trabajo en lugar de metodologías, debido a que estos utilizan las buenas prácticas de las metodologías, sin necesidad de ser una camisa de fuerza.

Por ejemplo, una desventaja de la metodología Lean es que no minimiza la administración, la dirección y el control del propietario, lo que se ha identificado como una de las principales causas de los intentos fallidos de implementar Lean en todas las organizaciones (Rivera \& Kashiwagi, 2016).

Por otra parte, una de las características que han impulsado Agile como una de las metodologías más utilizadas últimamente es que divide un proyecto en componentes más pequeños, utiliza la asociación entre todas las partes interesadas y las lecciones aprendidas se pueden implementar rápidamente en los otros componentes del proyecto (Rivera \& Kashiwagi, 2016).

A partir de un análisis realizado a las características reconocidas como ventajas de cada una de las tres metodologías estudiadas en el contenido de este documento, se define qué modelos integrados se pueden aplicar en la gestión de proyectos, como ejemplo en el desarrollo de software, señalando que un modelo puede integrar el enfoque de DT publicado por la Organización Internacional para la Estandarización (ISO), Lean y Scrum, este último entendido como metodología ágil, que también compone el modelo más utilizado recientemente.

En la construcción de un marco de trabajo que reúna bondades de Design Thinking, Lean y Agile (figura 2), se extraen para formar la primera parte del modelo las utilidades de Design Thinking, en cuanto a la identificación del problema a partir de la interacción con el cliente, en los siguientes pasos: 1) localización de problemas, 2) observación, 3) visualización y creación de sentido, 4) ideación, 5) creación de prototipos y pruebas, y 6) pruebas de viabilidad (Glen et al., 2015). Desde Lean, se extraen sus tres últimas fases, que serán implementadas para analizar, mejorar y controlar la construcción e impacto del proyecto; por otra parte, de Agile contribuye con las mejores prácticas para construcción y mejora de productos, entendidas como ceremonias de reunión efectivas, cada una con un objetivo distinto, el desarrollo de un MPv que genere valor permanentemente, y la modificación del trabajo que se debe realizar de acuerdo con los cambios en las externalidades del proyecto, es decir, la ausencia de un plan de trabajo a largo plazo definido, lo que permite una mayor respuesta al cambio de alcance. 
Figura 2. Modelo de integración Design Thinking, Lean y Agile

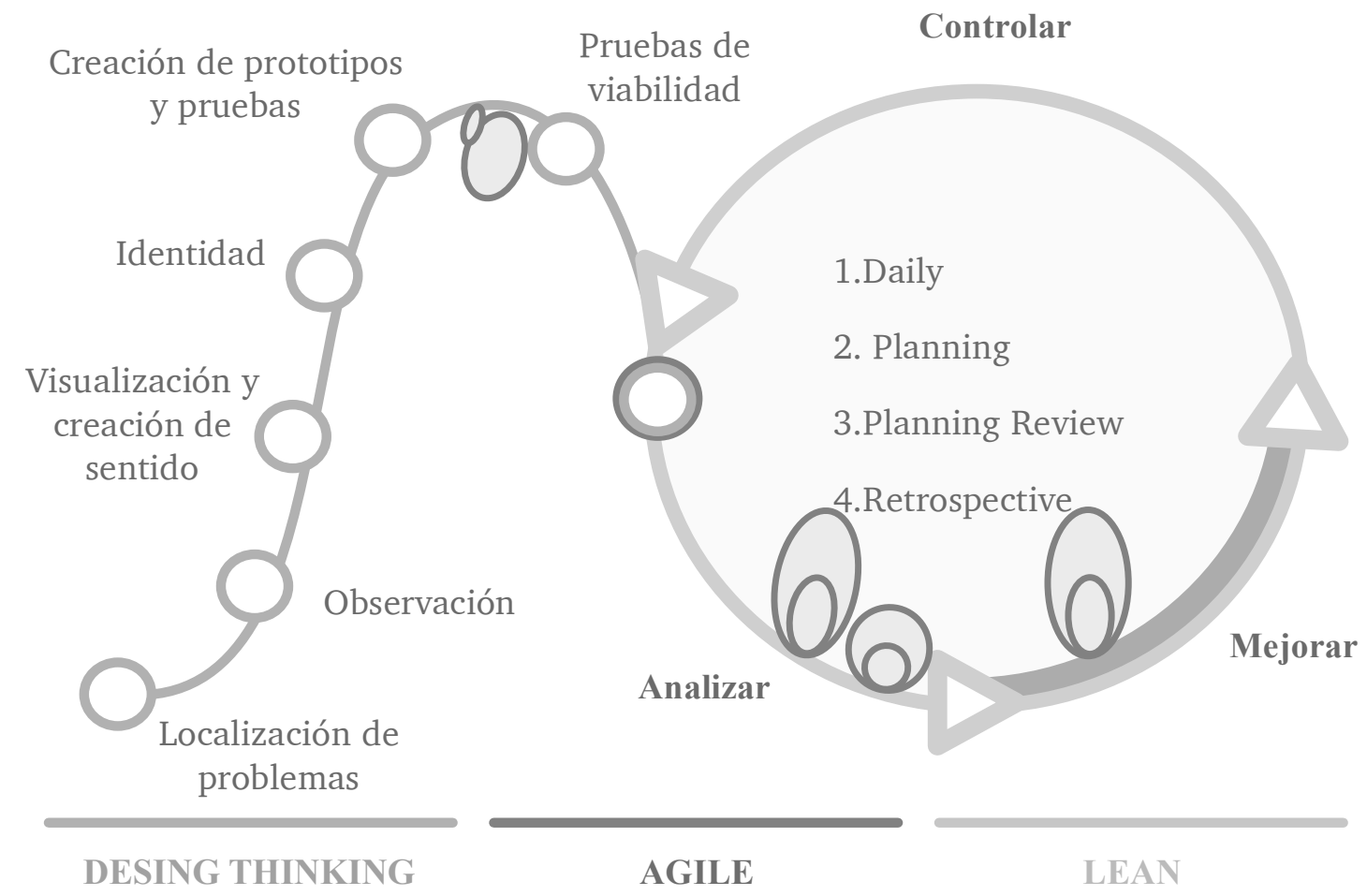

Fuente: adaptada de De Jonge (2019).

Si bien se han realizado aproximaciones a un modelo integrado de estas tres metodologías, la diferencia de esta propuesta es que no es de obligatoriedad la aplicación de todos los pasos, se pueden omitir de acuerdo con la forma como se decida adaptar el marco de trabajo a cada proyecto. Este marco de trabajo en sí permite aumentar la capacidad de respuesta al cambio, lo que es muy común en el contexto actual por el constante cambio al que se ven expuestas las compañías.

\section{CONCLUSIONES}

La gestión de proyectos ha sido cuestionada por muchas de sus entregas fuera de tiempo o con sobrecostos astronómicos; según cifras, el 60\% de los proyectos presenta un control de cambios, o su presupuesto fue mal estimado y se debe ejecutar más para obtener el mismo alcance, o se estima mal en cuanto a tiempos y el cronograma no se cumple, generando poca credibilidad a partir de la insatisfacción del cliente.

Por lo anterior, esta investigación, a partir de la definición de gestión de proyectos, Design Thinking, Lean y Agile, propuso un modelo muy general de trabajo que integra lo que se considera mejor de las tres formas de trabajo para la gestión de proyectos. Durante este recorrido, primero, se descubrió y sustentó la importancia y el papel trascendental de contar con una PMO, en el despliegue del conocimiento en torno a las mejores prácticas y esquemas de trabajo organizacionales para la gestión de proyectos.

Si bien cada una de estas tres tienen bondades propias y existen ejemplos de integración de estas como marcos de trabajo para diversas aplicaciones, no se identificó 
un marco de trabajo claro definido o un ejemplo de implementación de un proyecto que integre Design Thinking, Lean y Agile al mismo tiempo.

La integración de estos tres conceptos -Design Thinking, Lean y Agile- bajo un solo marco de trabajo o conjunto de buenas prácticas es relevante, porque las tres brindan cualidades distintas como orientación al cliente y generación de valor permanentemente, lo que hace interesante probarlas reunidas en un solo esquema; sin embargo, posterior a este documento es preciso implementar el modelo aquí propuesto en un proyecto, para definir el detalle de las herramientas, indicadores, roles, ventajas, desventajas, entre otros, y capturar sus beneficios cuantitativa y cualitativamente.

El impacto del marco de trabajo propuesto en la gestión de proyectos a cargo de las PMO es la obtención permanente de valor, dada por las entregas contantes durante la construcción y alineadas con los requerimientos del cliente, que fueron garantizadas por las etapas de Design Thinking. En conclusión, Design Thinking facilitará todo el trabajo previo y de levantamiento de información, garantizado el entregable bien definido a las condiciones iniciales, mientras que Lean y Agile funcionan garantizando la construcción eficiente, retroalimentación permanente y alta respuesta a los cambios del entorno.

\section{REFERENCIAS}

AlManei, M., Salonitis, K., \& Tsinopoulos, C. (2018). A conceptual lean implementation framework based on change management theory. Procedia CIRP, 72, 1160-1165. DoI: https://doi.org/10.1016/j.procir.2018.03.141

Carneiro, L. B., Silva, A. C. C. L. M., \& Alencar, L. H. (2018). Scrum Agile Project Management Methodology Application for Workflow Management: A Case Study. 2018 IEEE International
Conference on Industrial Engineering and Engineering

Management, 938-942. DoI: https://doi.org/10.1109/ IEEM.2018.8607356

Chou, D. C. (2018). Applying design thinking method to social entrepreneurship project. Computer Standards \&̊ Interfaces, 55, 73-79. DoI: https://doi.org/10.1016/j. csi.2017.05.001

De Jonge, M. (2019). Serious Scrum [Serious Scrum]. Recuperado de Serious Scrum website: https:// medium.com/serious-scrum/combine-design-thinking-lean-startup-and-agile-beware-of-waterfall-indisguise-46b230f536c9

Girgenti, A., Pacifici, B., Ciappi, A., \& Giorgetti, A. (2016). An Axiomatic Design Approach for Customer Satisfaction through a Lean Start-up Framework. Procedia CIRP, 53, 151-157. DOr: https:// doi.org/10.1016/j.procir.2016.06.101

Glen, R., Suciu, C., Baughn, C. C., \& Anson, R. (2015). Teaching design thinking in business schools. The International Journal of Management Education, 13(2), 182-192. Dor: https://doi.org/10.1016/j. ijme.2015.05.001

Joslin, R., \& Müller, R. (2015). Relationships between a project management methodology and project success in different project governance contexts. International Journal of Project Management, 33(6), 1377-1392. DoI: https://doi.org/10.1016/j.ijproman.2015.03.005

Lester, E. I. A. (2017). Project Definition. En Project Management, Planning and Control (pp. 1-5). Dor: https:// doi.org/10.1016/B978-0-08-102020-3.00001-2

Monteiro, A., Santos, V., \& Varajão, J. (2016). Project Management Office Models - A Review. Procedia Computer Science, 100, 1085-1094. DoI: https://doi. org/10.1016/j.procs.2016.09.254 
Morris, P. W. (2009). Implementing Strategy Through Project Management: The Importance of Managing the Project Front-end. En T, M. Williams et al. (Ed.), Making Essential Choices with Scant Information (pp. 36-67). London: Palgrave Macmillan. DoI: https://doi.org/10.1057/9780230236837_3

Papadopoulos, G. (2015). Moving from Traditional to Agile Software Development Methodologies Also on Large, Distributed Projects. Procedia - Social and Behavioral Sciences, 175, 455-463. Dor: https:// doi.org/10.1016/j.sbspro.2015.01.1223

Paton, S., \& Andrew, B. (2019). The role of the Project Management Office (PMO) in product lifecycle management: A case study in the defence industry. International Journal of Production Economics, 208, 43-52. DoI: https://doi.org/10.1016/j.ijpe.2018.11.002

Pereira, J. C., \& Russo, R. de F. S. M. (2018). Design Thinking Integrated in Agile Software Development: A Systematic Literature Review. Procedia Computer Science, 138, 775-782. DoI: https://doi. org/10.1016/j.procs.2018.10.101

Powell, M., \& Young, J. (2007). The project management support office. EnP. Morris \& J. Pinto, (Eds.), The Wiley Guide to Project Control (pp. 937-969). New Jersey: John Wiley and Songs.

Radujković, M., \& Sjekavica, M. (2017). Project Management Success Factors. Procedia Engineering, 196, 607-615. DoI: https://doi.org/10.1016/j. proeng.2017.08.048

Rasnacis, A., \& Berzisa, S. (2017). Method for Adaptation and Implementation of Agile Project Management Methodology. Procedia Computer Science, 104, 43-50. DoI: https://doi.org/10.1016/j.procs.2017.01.055

Rivera, A., \& Kashiwagi, J. (2016). Identifying the State of the Project Management Profession.
Procedia Engineering, 145, 1386-1393. DoI: https:// doi.org/10.1016/j.proeng.2016.04.204

Samset, K., \& Volden, G. H. (2016). Front-end definition of projects: Ten paradoxes and some reflections regarding project management and project governance. International Journal of Project Management, 34(2), 297-313. DoI: https://doi.org/10.1016/j.ijproman.2015.01.014

Silva, Sandra. E. P., Calado, R. D., Silva, M. B., \& Nascimento, M. A. (2013). Lean Startup applied in Healthcare: A viable methodology for continuous improvement in the development of new products and services. IFAC Proceedings Volumes, 46(24), 295-299. DoI: https://doi.org/10.3182/201309113-BR-3021.00054

Soltan, H., \& Mostafa, S. (2015). Lean and Agile Performance Framework for Manufacturing Enterprises. Procedia Manufacturing, 2, 476-484. https://doi. org/10.1016/j.promfg.2015.07.082

Špundak, M. (2014). Mixed Agile/Traditional Project Management Methodology - Reality or Illusion? Procedia -Social and Behavioral Sciences, 119, 939-948. DoI: https://doi.org/10.1016/j.sbspro.2014.03.105

Tenera, A., \& Pinto, L. C. (2014). A Lean Six Sigma (LSS) Project Management Improvement Model. Procedia - Social and Behavioral Sciences, 119, 912-920. DOI: https://doi.org/10.1016/j.sbspro.2014.03.102

Volkova, T., \& Jãkobsone, I. (2016). Design thinking as a business tool to ensure continuous value generation. Intellectual Economics, 10(1), 63-69. Dor: https:// doi.org/10.1016/j.intele.2016.06.003 